\title{
Computational Identification of Tumor Anatomic Location Associated with Survival in 2 Large Cohorts of Human Primary Glioblastomas
}

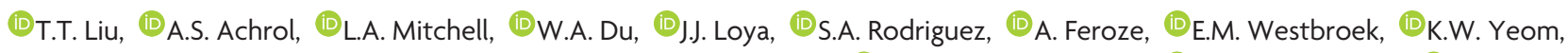
(1)J.M. Stuart, S.D. Chang, (1) G.R. Harsh IV, and D.D.L. Rubin

\begin{abstract}
BACKGROUND AND PURPOSE: Tumor location has been shown to be a significant prognostic factor in patients with glioblastoma. The purpose of this study was to characterize glioblastoma lesions by identifying MR imaging voxel-based tumor location features that are associated with tumor molecular profiles, patient characteristics, and clinical outcomes.
\end{abstract}

MATERIALS AND METHODS: Preoperative T1 anatomic MR images of 384 patients with glioblastomas were obtained from 2 independent cohorts ( $n=253$ from the Stanford University Medical Center for training and $n=131$ from The Cancer Genome Atlas for validation). An automated computational image-analysis pipeline was developed to determine the anatomic locations of tumor in each patient. Voxelbased differences in tumor location between good (overall survival of $>17$ months) and poor (overall survival of $<11$ months) survival groups identified in the training cohort were used to classify patients in The Cancer Genome Atlas cohort into 2 brain-location groups, for which clinical features, messenger RNA expression, and copy number changes were compared to elucidate the biologic basis of tumors located in different brain regions.

RESULTS: Tumors in the right occipitotemporal periventricular white matter were significantly associated with poor survival in both training and test cohorts (both, log-rank $P<.05$ ) and had larger tumor volume compared with tumors in other locations. Tumors in the right periatrial location were associated with hypoxia pathway enrichment and PDGFRA amplification, making them potential targets for subgroup-specific therapies.

CONCLUSIONS: Voxel-based location in glioblastoma is associated with patient outcome and may have a potential role for guiding personalized treatment.

ABBREVIATIONS: CEL = contrast-enhancing lesion; GBM = glioblastoma; GSEA = Gene Set Enrichment Analysis; KEGG = Kyoto Encyclopedia of Genes and Genomes; OS = overall survival; SVZ = subventricular zone; TCGA = The Cancer Genome Atlas; TFCE = threshold-free cluster enhancement

G ioblastoma (GBM, World Health Organization grade IV) is the most common primary brain cancer in adults. Despite decades of refinement, however, multimodal therapy of microsurgical resection, radiation, and chemotherapy results in median

Received May 27, 2015; accepted after revision August 2.

From the Stanford Center for Biomedical Informatics Research and Biomedical Informatics Training Program (T.T.L., D.L.R.), Department of Radiology (T.T.L., L.A.M., W.A.D., K.W.Y., D.L.R.), Stanford Institute for Neuro-Innovation and Translational Neurosciences (A.S.A.), Institute for Stem Cell Biology and Regenerative Medicine (A.S.A.), and Department of Neurosurgery (A.S.A., J.J.L., S.A.R., E.M.W., S.D.C., G.R.H.), Stanford University School of Medicine, Stanford, California; Department of Neurological Surgery (A.F.), University of Washington School of Medicine, Seattle, Washington; and Biomolecular Engineering (J.M.S.), University of California Santa Cruz, Santa Cruz, California.

T.T.L. and D.L.R. conceived and designed the study; G.R.H. and S.D.C. developed and maintained the Stanford GBM imaging database used as the training cohort T.T.L., A.S.A., L.A.M., W.A.D., J.J.L., S.A.R., A.F., E.M.W., K.W.Y., and S.D.C. acquired imaging data and performed annotations and preprocessing; T.T.L., J.M.S., and D.L.R. analyzed and interpreted the data; T.T.L. and D.L.R. wrote the manuscript; J.M.S., G.R.H., D.L.R., L.A.M., A.S.A., T.T.L., K.W.Y., and A.F. edited the manuscript; and all authors read and approved the manuscript. survival after diagnosis of only $12-15$ months. ${ }^{1}$ GBMs are heterogeneous with respect to genetic, molecular, and MR imaging characteristics. $^{2-4}$ Multiscale genomics and imaging analyses have revealed that GBMs with $I D H 1$ mutations, which have a favorable prognosis, ${ }^{5,6}$ tend to occur in brain regions different from those in which GBMs with wildtype IDH1 predominate, suggesting a potential prognostic role of tumor location in GBM. Prior imaging studies have also supported a relationship between GBM tumor location and clinical prognosis. ${ }^{7,8}$ Integrated multiscale analysis of MR imaging-based tumor location, patient characteristics, and genomic data may permit classification of patients with GBM

Please address correspondence to Daniel L. Rubin, MD, MS, Department of Radiology, 1201 Welch Rd, Stanford, CA 94305; e-mail: dlrubin@stanford.edu

- Indicates open access to non-subscribers at www.ajnr.org

三 Indicates article with supplemental on-line appendix and tables.

Indicates article with supplemental on-line photo.

http://dx.doi.org/10.3174/ajnr.A4631 
into subgroups with distinct genomic, tumor location, and clinical outcome characteristics.

The complexity and limited scalability of image-feature analysis has deterred inclusion of imaging data in multiscale integrated analysis. Radiogenomic studies that associate molecular features with quantitatively assessed image features describing tumor shape and texture often lack information regarding tumor location, likely due to the challenging and different imagepreprocessing techniques required to obtain this information. To date, information about tumor location has predominantly been qualitatively determined by radiologists, who annotate the locations of the tumors in high-level anatomic terms (eg, temporal lobe and so forth). Such qualitative assessment of tumor location can be onerous and may be affected by interobserver variability, lack of reproducibility, and scalability. In addition, qualitative assessment of tumor location has limited spatial granularity and fails to use the full resolution of MR imaging data available at the voxel level. A large-scale analysis of brain tumor image data at the voxel level could provide more anatomic detail compared with conventional qualitative approaches. Voxel-based image analysis linking the MR imaging appearance of GBM to patient survival has previously been undertaken in 1 study, ${ }^{9}$ but the results were not evaluated in an independent validation dataset and the study lacked analysis of imaging correlates with survival and tumor genomics.

The purpose of this study was to use computational imaging informatics methods to identify MR imaging voxel-based tumor location features and to seek associations of these with tumor molecular profiles, patient characteristics, and clinical outcomes. Our goal was to identify subtypes of GBMs on the basis of computationally derived tumor location that provide insight into prognosis and potentially guide more personalized therapy.

\section{MATERIALS AND METHODS \\ Patient Samples}

Gadolinium-based contrast-enhanced T1-weighted preoperative axial MR images of patients diagnosed with GBM and whose overall survival (OS) was known were acquired from 2 independent sources: the Stanford University Medical Center $(n=253)$ and The Cancer Genome Atlas (TCGA, $n=131) .{ }^{5,10}$ Institutional review board approval was obtained with informed consent for all patients. We selected the larger cohort for training and the small cohort for testing. The GBM cases in the training cohort were acquired by using 1.5T Signa Excite or Genesis Signa or Signa HDx MRI scanners (GE Healthcare, Milwaukee, Wisconsin) (TE, $8-20 \mathrm{~mm}$; TR, $360-1800 \mathrm{~mm}$ ) with a section thickness of 2 or $5 \mathrm{~mm}$. The images for the TCGA cohort were collected from 4 institutions by using 1.5T or 3.5T Signa Excite (GE Healthcare), 1.5T Genesis Signa (GE Healthcare), 1.5T Intera (Philips Healthcare, Best, the Netherlands), and 1.5T or 3T Achieva (Philips Healthcare) or 1.5T Magnetom Vision (Siemens, Erlangen, Germany) MRI scanners, respectively (TE, 3-20 mm; TR, 15-3280 $\mathrm{mm}$ ) with section thicknesses in the range of 1-5 mm, as previously described, ${ }^{10}$ and were downloaded from the Cancer Imaging Archive (www.cancerimagingarchive. net). ${ }^{11}$ Patients were included if preoperative imaging scans and OS information were available. Patient-matched level 3 gene expression and copy number data were downloaded from the TCGA data portal (https://tcga-data.nci.nih.gov/tcga/ dataAccessMatrix.htm).

\section{Image-Processing Pipeline to Extract Tumor Locations}

We derived voxelwise tumor localization on the basis of a common brain coordinate space for the 2 patient cohorts as described below (Fig 1A). First, an ROI was drawn to delineate the whole tumor, which includes the contrast-enhancing portion of the lesion (CEL) and the central necrosis on each axial section of the T1-weighted images in OsiriX imaging software (http:// www.osirix-viewer.com), and it was confirmed by a board-certified neuroradiologist (L.A.M.). Similarly, a necrosis ROI in the center of a tumor was defined as the nonenhancing, low-signalintensity region surrounded by a rim of enhancing tumor region on T1-weighted MR imaging. Tumor volume was computed in OsiriX. ${ }^{12}$ An automated image-processing pipeline was then developed to align all T1-weighted imaging volume to a common coordinate space in 3D Slicer (http://www.slicer.org) as follows: Briefly, a skull-stripping algorithm was applied to each T1weighted imaging volume to extract cerebral tissue, followed by affine registration to a $1.0-\mathrm{mm}$ isotropic brain atlas (Montreal Neurological Institute 152) by using a mutual information algorithm with a 12- $d f$ transformation. ${ }^{13,14}$ The same transform was used to align the ROI volume to the same Montreal Neurological Institute atlas coordinate space. The resulting registered T1weighted imaging and registered tumor binary ROI volumes were confirmed by consensus of 3 readers (T.T.L., A.S.A., L.A.M.).

\section{Training and Validation Data Cohorts}

To identify regions significantly associated with poor survival, we used the Stanford University Medical Center cohort $(n=253)$ for learning and the TCGA cohort $(n=131)$ as an independent validation set $($ Fig $1 B)$. The training cohort was stratified into poor, medium, and good overall survival groups defined by 2 cutoffs: $<11$ months and $>17$ months, which were 3 months below and above the median overall survival of 14 months, respectively (Fig $1 B$ ). The middle group was not assessed in the analyses to ensure a clear distinction between good and poor survival. We plotted the tumor locations across each group of patients in the training cohort as frequency heat maps, in which common events of tumor occurrence are shown as "hot" and less frequent events as "cooler" areas (Fig $1 B)$.

Voxels significantly associated with poor survival in classifying the Stanford University Medical Center training cohort were then used to dichotomize patients in the validation TCGA cohort (Fig $1 B)$. Lesions in the validation TCGA cohort having a nonzero number of voxels falling within the poor survival regions were classified as group I lesions. All other lesions were classified as group II lesions. The analysis was implemented in a Matlab script R2012a (MathWorks, Natick, Massachusetts). The survival difference between the 2 groups was evaluated by using the KaplanMeier survival analysis implemented in the survival package in $\mathrm{R}$ programming language (www.r-project.org).

\section{Statistical Analysis of Voxels Associated with Outcome}

To identify voxels significantly associated with poor survival, we applied the threshold-free cluster enhancement (TFCE) method 


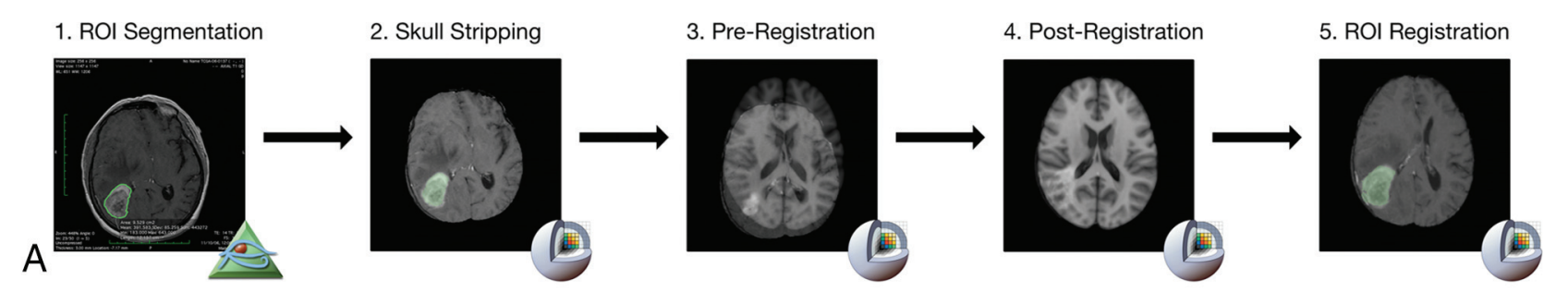

Algorithm training on SUMC cohort

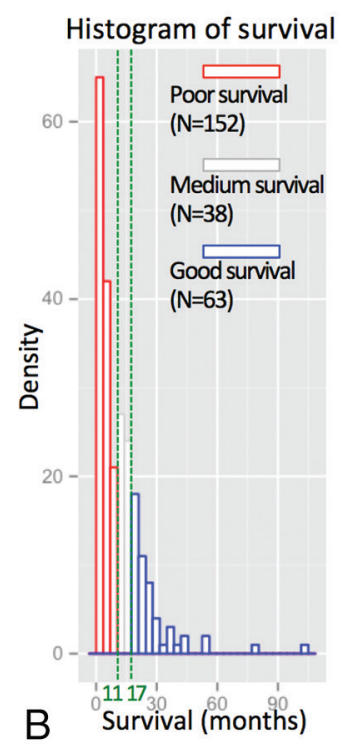

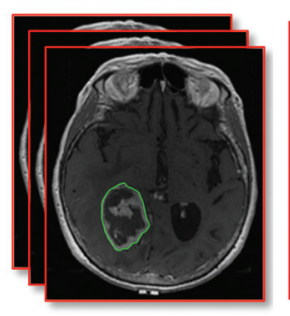

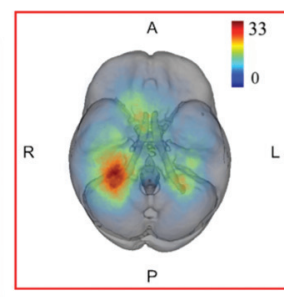

Poor survival ( $\leq 11$ months)
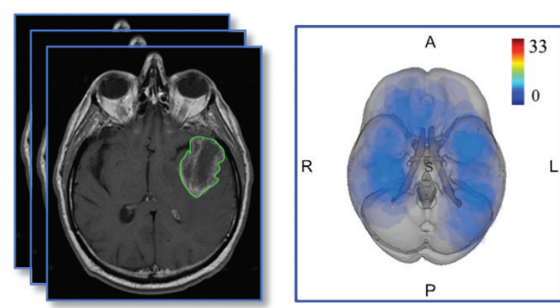

Good survival ( $\geq 17$ months)
Algorithm validation using TCGA cohort Uncategorized patients

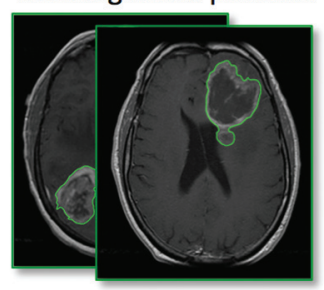

Prognostic imaging features (voxels)

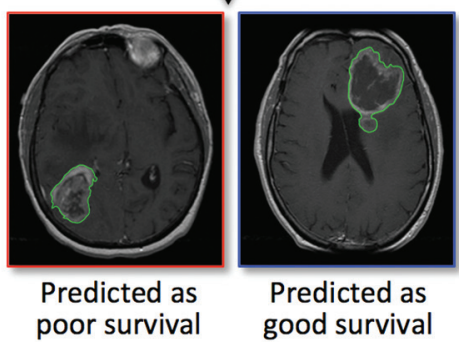

FIG 1. An overview of the image-processing pipeline and model training and validation procedure to identify locations associated with survival. A, The image-processing pipeline is applied to both training (Stanford University Medical Center) and validation (TCGA) cohorts. B, Algorithm training identifies anatomic regions associated with survival, which is validated in the TCGA cohort. The training algorithm using the thresholdfree cluster enhancement method takes as the input group labels dichotomized by survival outcome and the superimposed tumor heat map of the Stanford University Medical Center patient cohort analyzed in the image-processing pipeline; the pipeline outputs anatomic regions significantly associated with the 2 survival groups, which are used to classify the TCGA validation set into a poor survival group and a good survival group on the basis of tumor regions present or absent in the prognostic region.

with parameters optimized for 3D imaging volume implemented in FSL, Version 5.05 (http://www.fmrib.ox.ac.uk/fsl/), on a Linux system comparing the 2 clinical phenotypes (poor-versus-good survival). ${ }^{15}$ TFCE takes as input a cohort of patients' tumor ROI volumes and their binary survival group labels stratified by survival and computes a $P$ value for each voxel associated with each group. A total of 100 permutations were performed to correct for multiple comparisons and control for a family-wise error with a false discovery rate $<.05$. To evaluate the robustness of the results, we explored 286 pairs of lower and upper cutoffs in the overall survival at 3-15 months and 15-36 months to determine good and poor survival groups, respectively. The 286 pairs of cutoffs included the survival cutoffs defined in Ellingson et al. ${ }^{9}$ Each combination of survival cutoffs was used to define a poor survival group and a good survival group and was run for the prognostic region sensitivity analysis (On-line Appendix).

\section{Molecular and Genetic Analysis}

The Significance Analysis of Microarrays method ${ }^{16}$ was run on the microarray expression of the 2 identified patient groups (group I: tumors in the right periatrial location versus group II: tumors not in the right periatrial location) to compute a test sta- tistic for each gene, measuring the strength of association with the location groups to create a ranked list of all genes. The preranked gene list and previously curated gene set data base ${ }^{17}$ were used as the input of the Gene Set Enrichment Analysis (GSEA; http:// www.broad.mit.edu/gsea) to compute molecular enrichment between the 2 GBM location groups. Top enriched gene sets with a family-wise error rate $P$ value $<.05$ were reported and further confirmed by conducting the single-sample GSEA on the related pathway from the Kyoto Encyclopedia of Genes and Genomes (KEGG) data base, ${ }^{18}$ as previously described. ${ }^{4}$ The Significance Analysis of Microarrays was also run on log2 copy number data between the 2 location groups to identify amplified genes in each image location group by using the samr package in $\mathrm{R}$ (http://statweb.stanford.edu/ tibs/SAM/). ${ }^{16}$

\section{Known Clinical Variables}

Log-rank tests were used for categoric GBM clinical variables, including age younger than 64 years, sex, extent of surgical resection (biopsy, subtotal resection, or gross total resection) (available for 24 patients), and categoric tumor volume groups (large: top 30\%; intermediate: middle $40 \%$; and small: bottom $30 \%)$. The survival analysis was performed by using the sur- 
Table 1: Summary of clinical variables in the training cohort

\begin{tabular}{lccc}
\hline & \multicolumn{3}{c}{ Survival Groups } \\
\cline { 2 - 4 } & Poor (OS $\leq 11 \mathrm{mo})$ & Medium (11 < OS < 17 mo) & Good (OS $\geq 17 \mathrm{mo})$ \\
\hline Total No. & 152 & 38 & 63 \\
Median age (yr) & 69.0 & 57.2 & 59.5 \\
\% Male & 61.2 & 60.5 & 57.1 \\
Median survival (mo) & 4.1 & 14.1 & 21.2 \\
Mean survival (mo) & 4.8 & 14.0 & 26.3 \\
Mean CEL volume (cm ${ }^{3}$ ) & 34.1 & 34.0 & 28.9 \\
STR/GTR/biopsy only (No.) & $53 / 13 / 86$ & $17 / 8 / 13$ & $17 / 36 / 10$ \\
\hline
\end{tabular}

Note:-STR indicates subtotal resection; GTR, gross total resection.

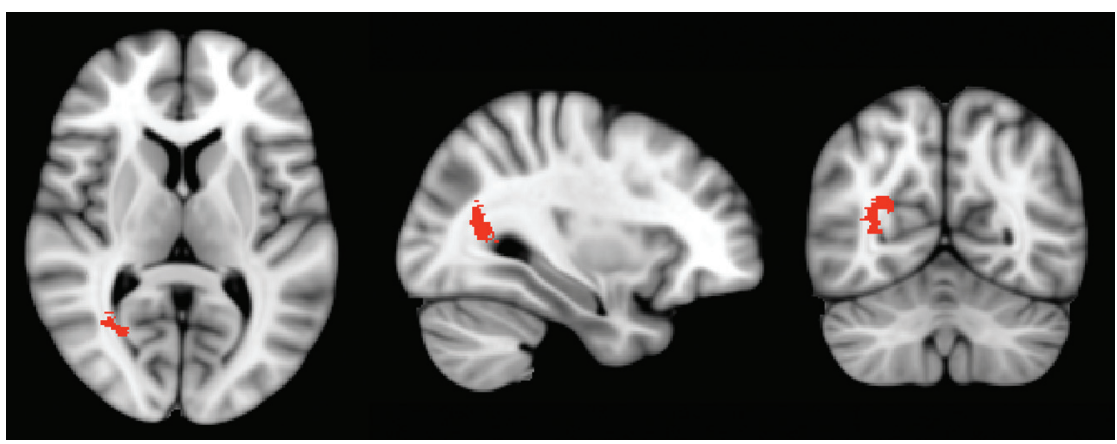

FIG 2. Axial, sagittal, and coronal section views of the region associated with poor survival in the training cohort (false discovery rate, $P<.05$ ). The cluster of voxels associated with poor survival was localized in the occipitotemporal periventricular white matter in the right hemisphere (right periatrial).

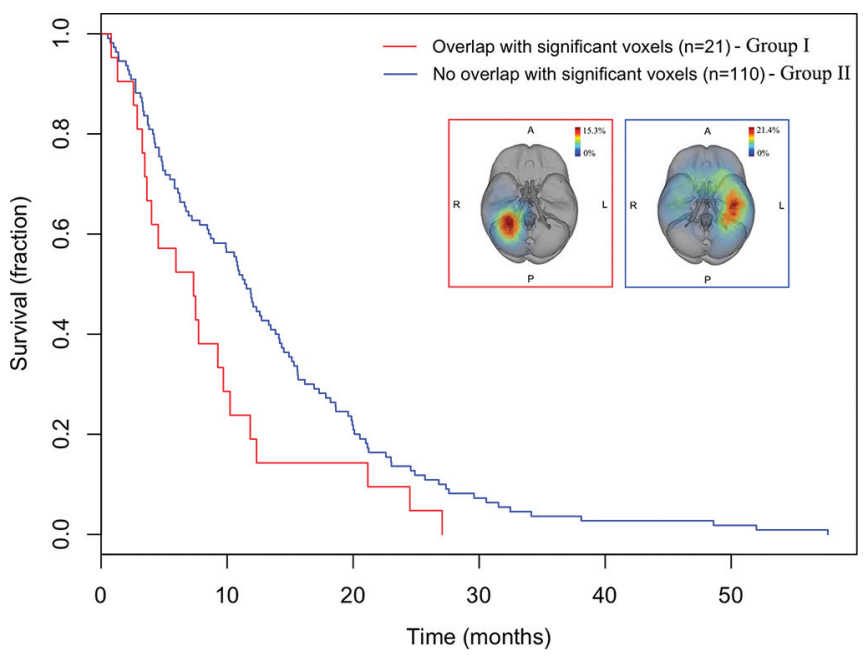

FIG 3. Kaplan-Meier survival curves of patients with GBMs depict decreased overall survival in TCGA patients with an overlap (group I) versus nonoverlap (group II) with the voxels significantly associated with survival identified from the training cohort in the test cohort (log-rank test, $P=$ .034).

vival package in $\mathrm{R}$ programming language. Significant variables in the univariate models $(P<.05)$ were included in the multivariate Cox proportional hazards regression model. The percentage of necrosis volume in the whole-tumor volume was available for 91 patients in the TCGA cohort.

\section{RESULTS}

\section{Clinical Characteristics of the Training Cohort}

An overview of image analysis and prognostic model construction is summarized in Fig 1. Clinical characteristics of the 3 survival groups in the training cohort are summarized in Table
1, and known clinical prognostic markers were confirmed by univariate analysis. Younger age was associated with better survival (age younger than 64 years, OS $=13.7$ versus age older than or equal to 64 years, 9.5 months, log-rank $P<.002$ ). More extensive surgical resection was associated with better overall survival (log-rank $P=$ $0)$, with gross total resection associated with longest average overall survival (22.5 months) compared with those patients with a subtotal resection (11.1 months) or those undergoing biopsy only (6.5 months). In contrast, tumor volume was not significantly associated with survival (hazard ratio $=$ $1, P=.58)$.

\section{Right Deep White Matter Tumors Are Associated with Poor Prognosis} The heat maps showing the frequency of tumor occurrence revealed that the poor and good survival groups had distinct imaging location phenotypic trends: The poor survival group had tumors in the right deep periventricular white matter region; in contrast, tumors associated with good survival occurred throughout the brains of patients without any particular anatomic region with high tumor frequency (Fig $1 B)$. TFCE analysis further confirmed that the voxels associated with poor prognosis (false discovery rate-adjusted $P<.05$ ) were in the occipitotemporal periventricular white matter in the right hemisphere ("right periatrial”) (Fig 2 and On-line Table 1), but no voxels were found to be significantly associated with tumors in the good survival group. Robustness analysis of 286 different pairs of lower and upper survival cutoffs at 3-15 months and 15-36 months showed that the results were largely invariant to the choice of survival cutoffs; the voxels associated with poor prognosis consistently occurred in the same region of the brain (On-line Figs 1 and 2). Among these 286 pairs included the survival cutoffs defined by Ellingson et al, ${ }^{9}$ no voxels were found to be significantly associated with survival, possibly a result of the small number of patients in the good survival group $(n=8)$.

\section{Prognostic Region Validated in the TCGA Dataset}

In the training cohort, tumors in the right periatrial location were significantly associated with poor survival $(\log -\operatorname{rank} P=$ .012 ). We then assessed whether this association generalized to 


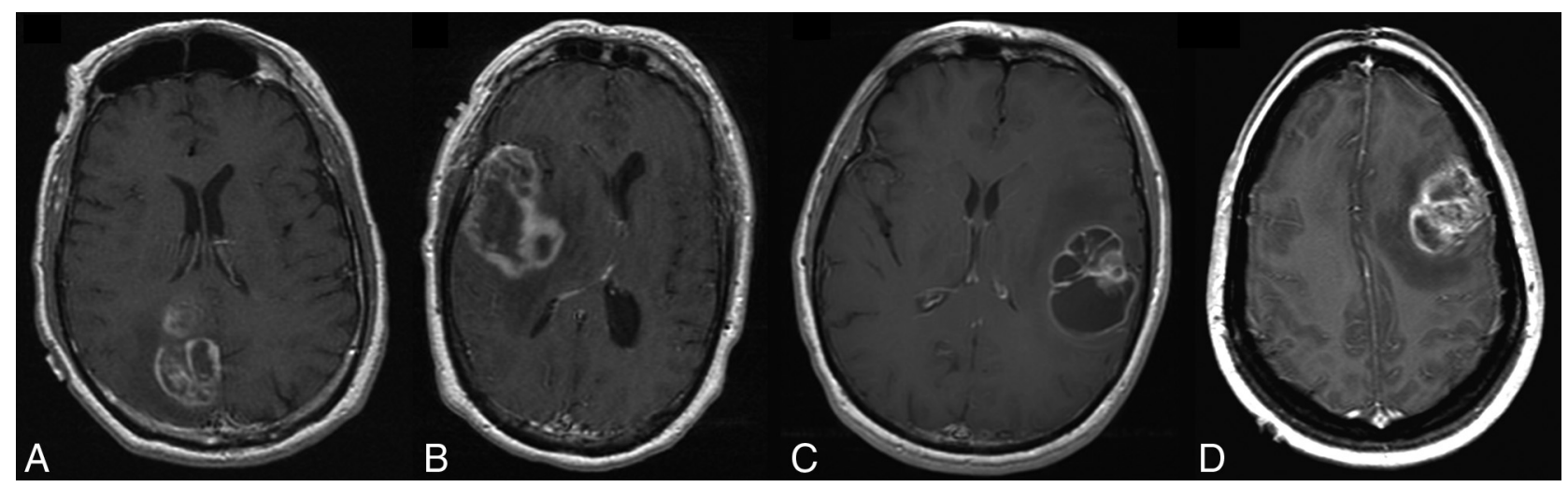

FIG 4. Axial postcontrast T1-weighted images of 4 patients from group II. A, A 69-year-old man with a right parietal GBM and an overall survival of 27 months. B, A 49-year-old man with a right temporal GBM and an overall survival of 25 months. C, A 63-year-old man with a left temporal GBM and an overall survival of 21 months. D, A 36-year-old woman with a left frontal GBM, an overall survival of 6 months, and the smallest tumor volume in the group of intermediate tumors.

Table 2: Univariate and multivariate Cox survival analysis of clinical variables

\begin{tabular}{|c|c|c|c|c|}
\hline & \multicolumn{2}{|c|}{ Univariate Cox } & \multicolumn{2}{|c|}{ Multivariate Cox } \\
\hline & $\mathrm{HR}(95 \% \mathrm{Cl})$ & $P$ Value & $\mathrm{HR}(95 \% \mathrm{Cl})$ & $P$ Value \\
\hline \multicolumn{5}{|l|}{ TCGA test cohort ${ }^{a}$} \\
\hline Age younger than $64 \mathrm{yr}$ & $0.19(0.26-0.55)$ & 4.le- $7^{\mathrm{b}}$ & $0.36(0.24-0.53)$ & $3.7 e-7^{b}$ \\
\hline Male sex & $1.05(0.73-1.50)$ & .79 & - & - \\
\hline \multicolumn{5}{|l|}{ CEL tumor volume $\left(\mathrm{cm}^{3}\right)$} \\
\hline Large & $1.68(1.10-2.54)$ & $.015^{\mathrm{b}}$ & $1.80(1.18-2.75)$ & $.0064^{\mathrm{b}}$ \\
\hline Intermediate & - & - & - & - \\
\hline Small & $0.90(0.58-1.37)$ & .62 & $1.11(0.71-1.71)$ & .65 \\
\hline Right laterality & $0.927(0.65-1.32)$ & .67 & - & - \\
\hline STR & $2.52(0.73-8.68)$ & .14 & - & - \\
\hline $\begin{array}{l}\text { Tumor location }=\text { right periatrial } \\
\text { location }\end{array}$ & $1.66(1.03-2.67)$ & $.036^{\mathrm{b}}$ & $2.0(1.01-2.64)$ & $.045^{\mathrm{b}}$ \\
\hline \multicolumn{5}{|l|}{$\begin{array}{l}\text { Restricted set of intermediate and } \\
\text { large tumors }{ }^{c}\end{array}$} \\
\hline Age younger than $64 \mathrm{yr}$ & $0.19(0.26-0.55)$ & 4.1e-7 & $0.46(0.30-0.72)$ & .00062 \\
\hline $\begin{array}{l}\text { Tumor location }=\text { right periatrial } \\
\text { location }\end{array}$ & $1.84(1.10-3.08)$ & .019 & $1.87(1.11-3.15)$ & .018 \\
\hline
\end{tabular}

Note:- - HR indicates hazard ratio; STR, subtotal resection.

${ }^{a}$ Age, CEL tumor volume, and tumor location were independently significant in multivariate analysis (overall $P<$

$3.52 \mathrm{e}-8)$. Surgical resection is subtotal resection or gross total resection, available for 24 cases in the TCGA test cohort.

${ }^{\mathrm{b}} \mathrm{P}$ value $<.05$, indicating the variable is significant.

' Tumor location remained significant in Cox analysis performed on the restricted set of intermediate and large tumors.

The right periatrial tumor location was significantly associated with poor survival, independent of age and tumor volume in the TCGA cohort $(P=.045)$ (Table 2).

The mean tumor volume in group I was 1.8 times the size of that in group II in the training cohort (Wilcoxon test, $P<1.5 \mathrm{e}-6)$ and 1.4 times that in the test cohort (Wilcoxon $P<.01$ ) (On-line Fig $3)$. However, the percentage of necrosis in the whole tumor was not significantly different between the 2 groups in the test cohort (Wilcoxon $P=.41$ ). The number of patients with GBMs in each hemisphere was about the same in both cohorts (124 left versus 118 right in the training cohort, and 70 left versus 61 right in the TCGA cohort), indicating the hotspot (highest frequency) of tumor occurrence found in the right periatrial location was associated with a larger

an independent validation set from TCGA samples. Patients with GBM of the TCGA cohort were dichotomized into 2 groups on the basis of whether their tumors overlapped the prognostic right periatrial region. The group with tumors overlapping the right periatrial location (group I) had significantly shorter overall survival than did those with tumors occurring in other locations (group II) (log-rank $P=.034$ ) (Fig 3). Examples of GBMs in group II are shown in Fig 4.

As in the training dataset, univariate survival analysis showed that age younger than 64 years $(\log$-rank $P<5 \mathrm{e}-7)$ was a significant prognostic factor in the test set, whereas sex $(\log$-rank $P=$ .79) and tumor laterality (left or right hemisphere) (log-rank $P=$ .67) were not significant in TCGA. We found that the surgical resection level, available for a small number of patients ( $n=24$ / 131), was not significantly associated with survival. The result may be due to the small number of patients available; thus, it was excluded in the multivariate analysis. Cox analysis on tumor volume categorized into 3 groups showed that large tumor volume was associated with poor survival (hazard ratio $=1.68, P=.015$ ). volume of the tumors in this region. Moreover, tumors in the right hemisphere were 1.5 and 1.1 times larger than those in the left hemisphere in the training (Wilcoxon $P<.0002$ ) and the test $(P=.42)$ cohorts, respectively, but tumor incidence in the right hemisphere did not confer a worse survival outcome in either cohort (both, log-rank $P>.05$ ), suggesting the specificity of the tumor localization being associated with prognosis.

To separate the effects of tumor volume and the right periatrial tumor location in predicting prognosis, we examined whether the right periatrial location still predicted worse prognosis when restricting the analysis to comparable tumor sizes. Because the right periatrial location is more relevant to larger tumors and to increase the statistical power of the analysis, we excluded small tumors $(n=38)$, combining the intermediate and large tumors $(n=93)$. When restricted to the combined subset of intermediate and large tumors, the right periatrial location was significantly associated with prognosis (hazard ratio $=1.84, \log -\operatorname{rank} P=.019$; Table 2). Intermediate-sized tumors may actually be more comparable in size with large tumors than with small tumors (Fig 4D). 
These results support tumor location contributing to clinical outcome, even after accounting for tumor size.

\section{Hypoxia Pathway Enrichment and Stem Cell Marker Amplification in Group I}

To identify significant transcriptomic alterations at the pathway level, the GSEA gene expression analysis revealed that 11 of the 37 significantly enriched pathways (family-wise error rate $P<.05$ ) in group I were hypoxia and HIF1A pathways (On-line Table 2). Single-sample GSEA, which generates a gene set enrichment score for each sample, further validated the hypoxia pathway being enriched in group I (Wilcoxon $P$ value $=0.0072$ ), compared with group II (On-line Fig 4). We did not find a significant association between the gene expression-based subtypes previously identified and the location subgroups (On-line Table 3).

Significance Analysis of Microarrays of log2 copy number data showed that several genes involved in stem cell (CHIC2, KIT, PDGFRA) and neural cell development (GSX2) (all false discovery rate $\mathrm{q}<0.05$ ) were significantly amplified in group I GBMs, compared with group II GBMs (On-line Table 4). ${ }^{19-22}$ The Fisher exact test by using discrete copy number alteration confirmed that PDGFRA $(P=.023)$, CHIC2 $(P=.016)$, GSX2 $(P=.025)$, and $\operatorname{KIT}(P=.025)$ genes were significantly amplified in group I tumors (On-line Tables 5-9). Most interesting, all 4 genes (KIT and PDGFRA encoding receptor tyrosine kinases, GSX2, and CHIC2) are colocalized on human chromosome band $4 \mathrm{q} 12 .{ }^{23}$ Other genes on the chromosome $4 \mathrm{q} 12$ (KDR, LNX1, REST) were also tested but were not significantly amplified in group I tumors.

To further tease out the confounding effect of tumor size from tumor location contributing to molecular alterations, we found that tumor volume was not significantly associated with PDGFRA amplification (Wilcoxon test, $P=.12$ ) or HIF overexpression (Spearman correlation coefficient $=-0.048, P=.62$ ). Singlesample GSEA confirmed significant upregulation of the hypoxia pathway in right periatrial tumors in intermediate-sized tumors only (Wilcoxon $P=.015$ ) and in the combined subset of intermediate and large tumors (Wilcoxon $P=.015$ ), respectively. The Fisher exact test by using discrete copy number data showed that the correlation between PDGFRA amplification and the right periatrial tumor location is significant in intermediate-sized tumors (Fisher exact test, $P=.018, n=53$ ) and in the combined set of intermediate and large-sized tumors (Fisher exact test, 2-tailed $P=.035 ; n=93$ ) (On-line Tables 5-9). These results provide further evidence that the genomic events are more associated with location than tumor size.

\section{DISCUSSION}

We have developed and applied an automated computational image-analysis pipeline that extracted quantitative tumor location information from MR images by aligning images from 384 cases of GBM to a common stereotactic space at a voxel level of resolution (Fig 1A). This allowed a more granular and quantitative characterization of tumor location than the predominant approach in prior work using qualitative assessment of anatomic location in general terms such as lobes and white matter. Using our voxel-based computational approach to studying tumor location in GBM, we have shown that a subgroup of GBMs localized in the occipitotemporal periventricular white matter of the right hemisphere (right periatrial) is associated with poor survival. We also showed that GBM tumors located in this region are characterized by large volume and a distinct molecular-alteration profile comprising hypoxia pathway enrichment and amplification of stem cell markers.

In related work, Ellingson et $\mathrm{al}^{9}$ computed the stereospecific frequency of tumor occurrence. Our work differs from that of Ellingson et al in a number of ways. First, the survival cutoffs for defining good and poor survival in Ellingson et al were 12 and 36 months, whereas ours were 11 and 17 months. Most patients with GBM have a poor prognosis, with a 3-year survival rate of 3\%$5 \% .{ }^{24}$ We performed survival analysis by using the patient groups produced by the cutoffs of Ellingson et al and found that no location voxels were significantly associated with survival (On-line Fig 2 ); this result is possibly due to the small proportion of patients in the good survival group $(n=8)$. Although it is interesting to study long-term survivors ( $\mathrm{OS}>36$ months), the good survival group we defined may be more relevant for GBM clinical treatment stratification.

A second difference between our work and that of Ellingson et $\mathrm{al}^{9}$ is that in contrast to an older voxelwise-based method they used for identifying tumor locations, our computational method applied a threshold-free cluster enhancement technique. ${ }^{15}$ The TFCE method provides better sensitivity and more interpretable output through enhancing voxel connectivity in a neighborhood than traditional voxel-based methods. A third difference between our work and that of Ellingson et al is that they reported results from a single cohort, whereas in our analysis, we trained on data from 1 institution and validated our results by using an independent public dataset consisting of data from multiple institutions (TCGA). A fourth difference is that unlike the prior work of Ellingson et al, we performed downstream molecular analysis, which allowed us to gain biologic insight into the GBM location associated with poor prognosis. Finally, Ellingson et al showed that tumor location in the right temporal lobe was generally more likely to be associated with shorter than with longer survival. Our analysis provides higher anatomic specificity by pinpointing a small region in the right periatrial deep white matter that appears to be associated with poor prognosis (On-line Table 1), consistent with previous qualitative analyses showing the relationship between poor survival and invasion of tumor cells into adjacent deep white matter tracts and the ependymal region. ${ }^{7,8}$

The association of invasive clinical phenotypes, location, and poor survival in the right periatrial location may relate to underlying neuroanatomy and tumor neurobiology. The relationship of tumor localization in the right hemisphere-rather than bilaterally-to survival may reflect differences in functional neuroanatomy. The eloquent brain regions, critical for language, motor, or sensory functions, are commonly found in the left hemisphere for most right-handed individuals. ${ }^{25}$ The fact that the right periatrial location is associated with poor prognosis may reflect a more delayed clinical presentation due to lack of eloquent functions in this region. This hypothesis is further supported by our observation that GBMs in the right hemisphere have larger CEL volume compared with those in the left hemisphere. Once tumors have grown larger, they are likely to have more extensive infiltration 
and tend to be more difficult to resect, which may confer worse prognosis. $^{26}$ The poorer prognosis for tumors occurring in the right periatrial white matter rather than the entire right cerebral hemisphere may reflect differences in tumor neurobiology. This finding concurs with prior reports of worse prognosis for tumors of the subventricular zone (SVZ) deep white matter (adjacent to the right periatrial location) that were based on qualitative assessment of tumor location. ${ }^{7,8}$ These studies have suggested that the SVZ region along the wall of the lateral ventricle, which contains neural stem cells, may be a niche environment conducive to the origin and/or development of primitive, aggressive glial tumors. ${ }^{7,9}$

Furthermore, our molecular profiling comparing the 2 location groups of the TCGA cohort demonstrated that PDGFRA is more likely to be amplified in group I. This finding is corroborated by previous reports that correlated glioma formation with increased expression of PDGFRA in neural stem cells of the SVZ. ${ }^{3,20}$ Other amplified genes (KIT and CHIC2) on the same chromosome 4q12 locus were also enriched in stem cell functions (On-line Tables 4-9). Thus, our results support the possibility that tumor neural stem cells may arise from the lateral ventricle of the SVZ region. Future work is needed to test this hypothesis.

Enrichment of the hypoxia pathway in group I is consistent with results of other studies that have shown that neural stem cells are maintained in a hypoxic niche. ${ }^{27,28}$ We also found significantly larger contrast-enhancing volume $(P=.004)$ of the right periatrial tumors relative to those without right periatrial involvement, potentially reflecting increased vascularity associated with elevated proangiogenic factors that are known to be inducible by hypoxia. ${ }^{29}$ Prior radiogenomic analysis has also shown that high contrast enhancement is associated with high expression of hypoxia-related genes. ${ }^{30}$ Notably, the percentage of central necrosis of the whole tumor volume in group I was not greater than that in group II $(P=.41)$; thus, the enrichment in the hypoxia pathway was not a result of greater central necrosis.

The outcome and differential molecular analyses performed on a subgroup of tumors with comparable sizes showed that the right periatrial tumors maintained significant associations with poor prognosis (log-rank $P=.019)$, with HIF1A and hypoxia pathway enrichment (Wilcoxon $P=.015$ ), and with PDGFRA amplification (Wilcoxon $P=.035$ ), independent of tumor volume. These results further imply that the poor prognosis of tumors may result from the stem cell niche in the right periatrial region enriched for HIF1A/hypoxia overexpression and PDGFRA amplification, ${ }^{31}$ rather than from the proliferative potential associated with larger tumor volume.

GBM is known to have extensive genetic heterogeneity. ${ }^{32,33}$ However, intratumoral heterogeneity was not directly addressed in this study because the genetic material was obtained from a single region of the tumor. Another limitation of our current study is that it lacked information on the precise biopsy locations from which the gene expression samples were taken (this information was not available to us). Validation by using such data, if available in the future, would strengthen our finding that the right periatrial location is correlated with stem cell marker amplification and hypoxia enrichment.

Our work advances knowledge in that by using a computa- tional pipeline with imaging informatics and radiogenomic analysis, we demonstrate that the anatomic location of GBM is a key prognostic variable, ${ }^{34}$ specifically for GBMs located in the right periatrial white matter. We also show that certain molecular alterations may be linked to specific tumor locations. The results from our work could help improve characterization of GBM subtypes and potentially guide more personalized treatment decisions. Our strategy for quantitative assessment of tumor locations, if applied to other anatomic location-specific diseases, may similarly identify connections among disease location, molecular signature, and patient outcome.

\section{CONCLUSIONS}

We conclude that voxel-based location in GBM is associated with patient outcome. Tumor location in the right occipitotemporal periatrial white matter is predictive of survival, independent of other known prognostic clinical variables, such as patient age and tumor volume. This result was further validated in an independent patient cohort. Using GBM anatomic location as the response variable, we were able to identify genomic enrichment associated with the imaging phenotype.

\section{ACKNOWLEDGMENTS}

We thank Drs Sanjiv Sam Gambhir, Jarrett Rosenberg, and Sofie Salama for helpful comments and discussion. We also gratefully acknowledge support for this study from Craig and Kimberly Darian and Carol Bade to Dr Steven Chang. We also appreciate Drs Rajan Jain and Laila Poisson for providing us the surgical resection information for the 24 TCGA patients from the Henry Ford Hospital.

Disclosures: Tiffany T. Liu—RELATED: Grant: National Institutes of Health (U01CA142555).* Joshua M. Stuart-RELATED: Grant: Agilent Fellowship for graduate student research in brain image-genomics. ${ }^{*}$ Daniel L. Rubin-RELATED: Grant: National Institutes of Health. * Money paid to the institution.

\section{REFERENCES}

1. Wen PY, Kesari S. Malignant gliomas in adults. N Engl J Med 2008; 359:492-507 CrossRef Medline

2. Larjavaara S, Mäntylä R, Salminen T, et al. Incidence of gliomas by anatomic location. Neuro Oncol 2007;9:319-25 CrossRef Medline

3. Dunn GP, Rinne ML, Wykosky J, et al. Emerging insights into the molecular and cellular basis of glioblastoma. Genes Dev 2012;26: 756-84 CrossRef Medline

4. Verhaak RG, Hoadley KA, Purdom E, et al; Cancer Genome Atlas Research Network. Integrated genomic analysis identifies clinically relevant subtypes of glioblastoma characterized by abnormalities in PDGFRA, IDH1, EGFR, and NF1. Cancer Cell 2010;17:98-110 CrossRef Medline

5. Brennan CW, Verhaak RG, McKenna A, et al. The somatic genomic landscape of glioblastoma. Cell 2013;155:462-77 CrossRef Medline

6. Yan H, Parsons DW, Jin G, et al. IDH1 and IDH2 mutations in gliomas. N Engl J Med 2009;360:765-73 CrossRef Medline

7. Lim DA, Cha S, Mayo MC, et al. Relationship of glioblastoma multiforme to neural stem cell regions predicts invasive and multifocal tumor phenotype. Neuro Oncol 2007;9:424-29 CrossRef Medline

8. Colen RR, Vangel M, Wang J, et al; TCGA Glioma Phenotype Research Group. Imaging genomic mapping of an invasive MRI phenotype predicts patient outcome and metabolic dysfunction: a TCGA glioma phenotype research group project. BMCMed Genomics 2014;7:30 CrossRef Medline

9. Ellingson BM, Lai A, Harris RJ, et al. Probabilistic radiographic atlas 
of glioblastoma phenotypes. AJNR Am J Neuroradiol 2013;34: 533-40 CrossRef Medline

10. Gutman DA, Cooper LA, Hwang SN, et al. MR imaging predictors of molecular profile and survival: multi-institutional study of the TCGA glioblastoma data set. Radiology 2013;267:560-69 CrossRef Medline

11. Clark K, Vendt B, Smith K, et al. The Cancer Imaging Archive (TCIA): maintaining and operating a public information repository. J Digit Imaging 2013;26:1045-57 CrossRef Medline

12. Rosset A, Spadola L, Ratib O. OsiriX: an open-source software for navigating in multidimensional DICOM images. J Digit Imaging 2004;17:205-16 CrossRef Medline

13. Johnson H, Harris G, Williams K. BRAINSFit: mutual information registrations of whole-brain 3D images, using the Insight Toolkit. Insight Journal 2007. http://hdl.handle.net/1926/1291. Accessed December 15, 2015

14. Fedorov A, Beichel R, Kalpathy-Cramer J, et al. 3D Slicer as an image computing platform for the Quantitative Imaging Network. Magn Reson Imaging 2012;30:1323-41 CrossRef Medline

15. Smith SM, Nichols TE. Threshold-free cluster enhancement: addressing problems of smoothing, threshold dependence and localisation in cluster inference. Neuroimage 2009;44:83-98 CrossRef Medline

16. Tusher VG, Tibshirani R, Chu G. Significance analysis of microarrays applied to the ionizing radiation response. Proc Natl Acad Sci U S A 2001;98:5116-21 CrossRef Medline

17. Subramanian A, Tamayo P, Mootha VK, et al. Gene set enrichment analysis: a knowledge-based approach for interpreting genomewide expression profiles. Proc Natl Acad Sci U S A 2005;102: 15545-50 CrossRef Medline

18. Kanehisa M, Goto S. KEGG: Kyoto encyclopedia of genes and genomes. Nucleic Acids Res 2000;28:27-30 CrossRef Medline

19. Jörnsten R, Abenius T, Kling T, et al. Network modeling of the transcriptional effects of copy number aberrations in glioblastoma. $\mathrm{Mol}$ Syst Boil 2011;7:486 CrossRef Medline

20. Jackson EL, Garcia-Verdugo JM, Gil-Perotin S, et al. PDGFR alphapositive B cells are neural stem cells in the adult SVZ that form glioma-like growths in response to increased PDGF signaling. $\mathrm{Neu}$ ron 2006;51:187-99 CrossRef Medline

21. Puputti M, Tynninen O, Sihto H, et al. Amplification of KIT, PDGFRA, VEGFR2, and EGFR in gliomas. Mol Cancer Res 2006;4: 927-34 CrossRef Medline

22. Méndez-Gómez HR, Vicario-Abejón C. The homeobox gene Gsx2 regulates the self-renewal and differentiation of neural stem cells and the cell fate of postnatal progenitors. PLoS One 2012;7:e29799 CrossRef Medline

23. Blom T, Roselli A, Tanner M, et al. Mutation and copy number analysis of LNX1 and Numbl in nervous system tumors. Cancer Genet Cytogenet 2008;186:103-09 CrossRef Medline

24. Krex D, Klink B, Hartmann C, et al; German Glioma Network. Longterm survival with glioblastoma multiforme. Brain 2007;130(pt 10): 2596-606 CrossRef Medline

25. Kim SS, McCutcheon IE, Suki D, et al. Awake craniotomy for brain tumors near eloquent cortex: correlation of intraoperative cortical mapping with neurological outcomes in $\mathbf{3 0 9}$ consecutive patients. Neurosurgery 2009;64:836-45; discussion 345-46 CrossRef Medline

26. Sanai N, Berger MS. Glioma extent of resection and its impact on patient outcome. Neurosurgery 2008;62:753-64; discussion 264-66 CrossRef Medline

27. Heddleston JM, Li Z, McLendon RE, et al. The hypoxic microenvironment maintains glioblastoma stem cells and promotes reprogramming towards a cancer stem cell phenotype. Cell Cycle 2009;8: 3274-84 CrossRef Medline

28. Li Z, Bao S, Wu Q, et al. Hypoxia-inducible factors regulate tumorigenic capacity of glioma stem cells. Cancer Cell 2009;15:501-13 CrossRef Medline

29. Kaur B, Khwaja FW, Severson EA, et al. Hypoxia and the hypoxiainducible-factor pathway in glioma growth and angiogenesis. Neuro Oncol 2005;7:134-53 CrossRef Medline

30. Diehn M, Nardini C, Wang DS, et al. Identification of noninvasive imaging surrogates for brain tumor gene-expression modules. Proc Natl Acad Sci U S A 2008;105:5213-18 CrossRef Medline

31. Li L, Candelario KM, Thomas K, et al. Hypoxia inducible factor-1 $\boldsymbol{\alpha}$ $(\mathrm{HIF}-1 \alpha)$ is required for neural stem cell maintenance and vascular stability in the adult mouse SVZ. J Neurosci 2014;34:16713-19 CrossRef Medline

32. Snuderl M, Fazlollahi L, Le LP, et al. Mosaic amplification of multiple receptor tyrosine kinase genes in glioblastoma. Cancer Cell 2011; 20:810-17 CrossRef Medline

33. Sottoriva A, Spiteri I, Piccirillo SG, et al. Intratumor heterogeneity in human glioblastoma reflects cancer evolutionary dynamics. Proc Natl Acad Sci U S A 2013;110:4009-14 CrossRef Medline

34. Sturm D, Witt H, Hovestadt V, et al. Hotspot mutations in H3F3A and IDH1 define distinct epigenetic and biological subgroups of glioblastoma. Cancer Cell 2012;22:425-37 CrossRef Medline 